This item was submitted to Loughborough's Research Repository by the author.

Items in Figshare are protected by copyright, with all rights reserved, unless otherwise indicated.

\title{
The role of perceived severity of disaster, rumination, and trait resilience in the relationship between rainstorm-related experiences and PTSD amongst Chinese adolescents following rainstorm disasters
}

\section{PLEASE CITE THE PUBLISHED VERSION}

http://dx.doi.org/10.1016/j.apnu.2017.06.003

\section{PUBLISHER}

(C) Elsevier

\section{VERSION}

AM (Accepted Manuscript)

\section{PUBLISHER STATEMENT}

This work is made available according to the conditions of the Creative Commons Attribution-NonCommercialNoDerivatives 4.0 International (CC BY-NC-ND 4.0) licence. Full details of this licence are available at: https://creativecommons.org/licenses/by-nc-nd/4.0/

\section{LICENCE}

CC BY-NC-ND 4.0

\section{REPOSITORY RECORD}

Quan, Lijuan, Rui Zhen, Benxian Yao, Xiao Zhou, and Dapeng Yu. 2019. "The Role of Perceived Severity of Disaster, Rumination, and Trait Resilience in the Relationship Between Rainstorm-related Experiences and PTSD Amongst Chinese Adolescents Following Rainstorm Disasters". figshare.

https://hdl.handle.net/2134/25920. 
The Role of Perceived Severity of Disaster, Rumination, and Trait Resilience in the Relationship Between Rainstorm-related Experiences and PTSD among Chinese Adolescents Following Rainstorm Disasters

Lijuan Quan $^{\text {a }}$, Rui Zhen ${ }^{\mathrm{b}}$, Benxian Yao ${ }^{\mathrm{a}}, \underline{\text { Xiao Zhou }}^{\mathrm{c} *}$, and Dapeng Yu${ }^{\mathrm{d}}$

a. College of Educational Science, Anhui Normal University, Anhui Wuhu 241000, China

b. Beijing Key Laboratory of Applied Experimental Psychology, School of Psychology, Beijing Normal University, Beijing 100875, China

c. I-Core Research Center for Mass Trauma; School of Social Work, Tel Aviv University, Tel Aviv 69978, Israel

d. Department of Geography, Loughborough University, Loughborough, Leicestershire, UK

\section{Corresponding:}

Xiao Zhou, Ph.D.

I-Core Research Center for Mass Trauma; School of Social Work

Tel Aviv University

Tel Aviv 69978, Israel

E-mail: zxzhouxiao111@gmail.com 


\section{Abstract}

This study examined the mediating role of perceived severity of trauma and rumination in the relationship between traumatic experiences and PTSD, and assessed the moderated role of resilience in this mediating process. Nine hundred and fifty-one adolescents were selected to complete a self-report questionnaire involving rainstormrelated experiences, perceived severity of trauma, rumination, resilience, and PTSD. The results found that rainstorm-related experiences had a positive effect on PTSD by perceived severity of disaster, or by rumination via perceived severity of disaster. Resilience buffered the relationship between rainstorm-related experiences and PTSD, but did not buffer the relation of rainstorm-related experiences to perceived severity of disaster and rumination. These findings indicated that rainstorm-related experiences may have an indirect effect on PTSD via cognitive activities, and these indirect paths were not buffered by resilience. A buffering effect only occurred in the direct paths from rainstorm-related experiences to PTSD.

Key words: rainstorm-related experiences, perceived severity of disaster, rumination, resilience, PTSD 
It has been documented that posttraumatic stress disorder (PTSD) is a common negative outcome amongst individuals following traumatic experiences. Studies have found that the prevalence of PTSD in a traumatized population following natural disasters ranges from $5 \%$ to $60 \%$ (Galea, Nandi, \& Vlahov, 2005). In China, these rates have been found to range from $6.5 \% \sim 82.6 \%$ (Cao, Wang, Cao, \& Zhang, 2015). Of particular importance is the finding that adolescents may report more severe PTSD symptoms than adults because of their susceptibility to trauma (Eth, 2008). For example, Ying et al.' (2015) study on adolescents following the Wenchuan earthquake found that $8.4 \%$ of individual's showed signs of PTSD. A study on adolescents who experienced a flood in southwestern Poland found that $18 \%$ of the participants met full diagnostic criteria for PTSD (Bokszczanin, 2007). Agustini, Asniar and Matsuo (2011) found that very severe to moderate PTSD symptoms occurred in $63.1 \%$ of 482 adolescent participants after the tsunami in Aceh.

These higher prevalence rates of PTSD have led to a recent interest amongst researchers to examine the risk factors for PTSD. Two meta-analyses have found that there are many risk factors for PTSD in adolescents such as traumatic exposure, perceived life threat, and other post-traumatic factors (Furr, Comer, Edmunds, \& Kendall, 2010; Trickey, Siddaway, Meiser-Stedman, Serpell, \& Field, 2012), wherein traumatic experiences might be a prerequisite for PTSD (Freedy, Resnick, \& Kilpatrick, 1992; Janoff-Bulman, 2010). Theoretically, traumatic experiences may challenge an individual's existing and stable belief system, leading them to form negative assumptions about their posttraumatic world and, in turn, elicit PTSD (Janoff-Bulman, 
2010). Studies have also suggested that the differences in the prevalence rates and severity of PTSD symptoms may depend on the degree of exposure to the traumatic experiences (Grubaugh, Zinzow, Paul, Egede, \& Frueh, 2011). Furthermore, several studies have found that traumatic experiences have positive effect on PTSD (Fan, Zhang, Yang, Mo, \& Liu, 2011; Ma et al., 2011).

Nevertheless, other studies have found that traumatic experiences do not necessarily directly lead to PTSD (Nygaard \& Heir, 2012; Zhou, Wu, Yuan, Chen, \& Chen, 2015), and therefore not all individuals who experience a trauma will necessarily develop PTSD following traumatic events (Wu, Zhou, Lin, \& Chen, 2015). A potential reason for these mixed findings may be attributed to the cognitive activities following a traumatic experience (Bomyea \& Lang, 2016; Janoff-Bulman, 2010; Joseph, Williams, \& Yule, 1997; Park, 2010; Ponnamperuma \& Nicolson, 2016). Studies on PTSD have found that the perceived severity of a trauma and rumination may be two important cognitive factors that lead an individual to experience distress (Blanchard et al., 1995; Cernvall et al., 2016; Delahanty, Raimonde, Spoonster, \& Cullado, 2003; Zhou \& Wu, 2016). Therefore, the aim of this study was to assess the role of perceived severity of disasters and levels of rumination in relationship between traumatic experiences and PTSD, and to elucidate the underlying cognitive processes of the occurrence of PTSD amongst adolescents after a rainstorm disaster.

\section{Perceived severity of disaster, Rumination, and PTSD}

The perceived severity of disasters involves the appraisal and associated cognitions regarding the severity of the disaster outcomes (e.g., evaluating the 
economic and health consequences following a natural disaster). The cognitive theory of stress and coping suggests that an individual's cognitive appraisal and coping process plays an important role in the resulting psychological outcomes (Lazarus \& Folkman, 1984). Based on this theory, the experience of a traumatic event can activate an individual's appraisal system, wherein people are primarily focused on the potential harm associated with a traumatic experience. When individual who has recently suffered a trauma evaluates and perceives the traumatic experience as being threatening, they may show more fear or worry than an individual who does not. This can lead to a conditioned fear of trauma-related cues (Jovanovic et al., 2009), wherein traumatic events may easily intrude into peoples' cognitive worlds (Fani et al., 2012) and elicit PTSD symptoms, particularly those related to re-experiencing the event(s). Thus, studies have reported that having a greater perceived severity of an experienced trauma was associated with more symptomatology associated with PTSD (Besser, Neria, \& Haynes, 2009; Girelli, Resick, Marhoefer-Dvorak, \& Hutter, 1986; Kemp, Rawlings, \& Green, 1991; Lee, Ahn, Jeong, Chae, \& Choi, 2014; Solomon, Mikulincer, \& Hobfoll, 1987). Taken together, it may be that the perceived severity of trauma may mediate the relationship between traumatic experiences and PTSD.

Another important predictive factor of PTSD is rumination, which refers to a mode of responding to distress that involves repetitively and passively focusing on symptoms of distress and on the possible causes and consequences of these symptoms (Nolen-Hoeksema, Wisco, \& Lyubomirsky, 2008). Janoff-Bulman (2010) claimed that the shattered world assumption, led by trauma severity, caused substantial internal 
disequilibrium before and after a trauma and, in turn, could cause a traumatized individual to immerse him/herself into the traumatic events and ruminate on them. Furthermore, rumination on traumatic events causes individuals to focus on the negative outcomes of traumatic events and, in turn, increases negative emotions (Blackburn \& Owens, 2016), which may ultimately lead to PTSD (Borders, Rothman, \& McAndrew, 2015; Chuen Yee Lo, Lau, Cheung, \& Allen, 2012). Thus, it was proposed that traumatic experiences may be indirectly associated with PTSD via rumination.

Although traumatic experiences may lead to more PTSD symptoms via the activation of one's perceptions of trauma severity or rumination, the complimentary role of the perceived severity of a disaster and rumination has seldom been examined. In fact, Lazarus and Folkman (1984) suggested that the appraisal of traumatic experiences might be associated with cognitive coping. That is, the perceived severity of trauma may be related to rumination because rumination can be considered as a way of coping following a traumatic event (Baschnagel, Gudmundsdottir, Hawk, \& Beck, 2009). Specifically, when traumatized people perceive a trauma as being of a higher severity, they also tend to perceive more psychological stress which, in turn, undermines their emotional ability to self-regulate, or the capacity to engage in self-control over one's behavior (Baumeister, Gailliot, DeWall, \& Oaten, 2006). This may lead traumatized individuals to focus their attention on negative thoughts and feelings, which can lead to the formation of negative self-schemas based on their traumatic experiences, and increase the likelihood of rumination (Michl, McLaughlin, Shepherd, \& Nolen- 
Hoeksema, 2013; Scher, Ingram, \& Segal, 2005). Taken collectively, it is likely that traumatic experiences may also have indirect effects on PTSD based on an individual's perceived severity of the trauma via rumination.

Nevertheless, it has been noted when examining the predictive factors of PTSD, individual characteristics and traits should not be ignored (Bonanno, 2004). The diathesis-stress model emphasizes that exposure to traumatic events interacts with individual characteristics and traits to produce trauma-related outcomes (McKeever \& Huff, 2003), wherein trait resilience may be an important individual characteristic (Besser \& Priel, 2010; Wagnild, 2009). Furthermore, studies have indicated that resilience may buffer the role of traumatic experiences in PTSD (Fincham, Altes, Stein, \& Seedat, 2009; Lee et al., 2014), but it is still unclear as to whether resilience buffers the underlying cognitive processes of PTSD following a traumatic event. Therefore, this study further assessed the potential moderating role of resilience in paths from traumatic experiences to PTSD.

\section{Moderated Role of Resilience}

Trait resilience refers to a positive personality trait that enables individuals to recover from adversity and to adapt, thrive and mature in the face of difficult or challenging circumstances (Friborg, Hjemdal, Rosenvinge, \& Martinussen, 2003; Liu, Wang, Zhou, \& Li, 2014). Resilient individuals are more likely to be hardy (Bonanno, 2005, 2008), wherein they tend to view potentially stressful situations as meaningful, have a continued commitment to their values and goals of life after stressful situations, believe that they have some control over their stressors, appraise stressors as changeable, 
and see change as a normal aspect of life (Jakšić, Brajković, Ivezić, Topić, \& Jakovljević, 2012; Nabizadeh \& Mahdavi, 2016). Therefore, after traumatic experiences, individuals with greater resiliency may perceive a traumatic event as being less severe. In contrast, individuals with lower resiliency may be inclined to perceive a traumatic event as being more severe.

In addition to hardiness, features of resilience include self-improvement, positive impact, coping and a sense of coherence (Tedeschi \& Calhoun, 2004). Resilient individuals have been found to be more likely to use creative exploration, relaxation, and optimistic thinking as ways of coping (Tugade \& Fredrickson, 2004). More importantly, Calhoun and Tedeschi's (2006) theory suggests that resilient individuals may look at traumatic experiences as being less aversive, and thus their beliefs may challenge less. The result being that individuals ruminate less in response to traumatic cues (Tedeschi \& Calhoun, 2004). Therefore, it is more likely highly resilient adolescents may engage in less rumination, than less resilient adolescents following a traumatic experience. That is, resilience may also buffer the role of traumatic experiences with respect to rumination.

Additionally, resilient trauma survivors show other characteristics such as selfesteem and optimism (Bonanno, 2005, 2008), this may permit people to persist in normal functioning despite a traumatic exposure (Bensimon, 2012), such that resilient individuals remain stable before, during and after their exposure to trauma (Bonanno, 2008). Therefore, higher resilience could protect traumatized individuals from posttraumatic negative outcomes, and reduce susceptibility to negative psychological 
outcomes (Pietrzak, Johnson, Goldstein, Malley, \& Southwick, 2009; Wingo et al., 2010), whereas individuals with comparatively low resiliency may be more inclined to perceive more distress. In other words, resilience may also buffer the role of traumatic experiences in PTSD (Dai et al., 2014; Lee et al., 2014).

\section{Current study}

The literature reviewed above suggests that the unique role of traumatic experiences, perceived severity of trauma, rumination, and resilience in predicting the onset of PTSD, but no studies have assessed their combined role in producing the symptoms of PTSD. In addition, theoretically, both perceived severity of trauma and rumination may mediate the relationship between traumatic experiences and PTSD, but the predictive utility of such relationships has not yet been evaluated in adolescent survivors of natural disasters. Moreover, the complimentary role of perceived severity of disasters and rumination in the relationship between traumatic experiences and PTSD has seldom been examined, which further limits our understanding of the underlying processes of PTSD. More importantly, although the potential moderating role of resilience in the relationship between traumatic experiences and PTSD had been identified (Ying, Wu, Lin, \& Jiang, 2014), the role of resilience in the relationship between traumatic experiences and cognitive factors (e.g., perceived severity of trauma, rumination) is still unclear. Finally, natural disasters, which are both unpredictable and uncontrollable traumatic events, may seriously destroy a victim's cognitions and beliefs (Ehlers \& Clark, 2000), such that a better understanding of the mediating and moderating factors represents a pressing need in terms of both decreasing PTSD and 
promoting adjustment amongst survivors following natural disasters.

Given this backdrop, the current study examined how traumatic experiences, perceived severity of trauma, rumination, and resilience were associated with PTSD amongst adolescents following a severe rainstorm. Specifically, a moderated serial multiple mediation model is hypothesized wherein rainstorm-related experiences may have an indirect effect on PTSD via the perceived severity of the disaster, or via rumination. We also predicted that the traumatic experiences may have an indirect effect on PTSD by the perceived severity of the disaster via rumination, and that resilience may moderate the paths from the rainstorm-related experiences to the perceived severity of trauma, rumination, and PTSD (see figure 1 for the theoretically hypothesized model)

\section{--Insert Figure 1--}

\section{Method}

\section{Participants and Procedures}

This study was approved by the Research Ethics Committee of Anhui Normal University and conducted with the permission of the principals of the participating schools. To recruit participants, we first chose the areas where rainstorms frequently occur in China, with total rainfall amounting to more than $200 \mathrm{~mm}$ in 24 hours according to the Chinese Meteorological Administration. Then, we selected several cities, such as Nanjing, Huainan, and Huangshan, in the middle-lower reaches of the Yangtze River and the Huai River in China (i.e., areas that have relatively high occurrences of rainstorm disasters). Next, we selected several middle schools in these 
areas with the approval of the principals of the participating schools. We then randomly selected several classes within these schools. In the selected classes, everyone who attended school on the date of the survey was recruited to participate. There were no exclusion criteria. Compensation was not provided. The purpose of the study and the voluntary nature of students' participation were highlighted before the survey, and written consent forms that emphasized the right of each participant to withdraw from the survey at any time were provided to participants. The researchers administered the questionnaire packets in the classroom settings without the teachers' presence. The participants first provided demographic information that included gender and age. They were then asked to complete measures that assessed rainstorm-related experiences, rumination, social support, and PTSD. After the questionnaire packets were completed, the participants were told that school psychologists or teachers were available to provide any psychological/counseling services that they might need. In this study, 951 middle school students participated in this investigation. $453(47.6 \%)$ were male and $490(51.6 \%)$ were female, and $8(0.8 \%)$ participants did not report gender. The mean age was $14.78(\mathrm{SD}=1.70)$ years old, with a range from 12.0 to 19.0 .

\section{Measures}

Rainstorm-related experiences. Adolescents' rainstorm-related experiences were assessed by using our self-developed questionnaire. To date, there is no wellestablished measure for rainstorm-related experiences, so we developed one by referring to the developing process of the Hurricanes-related Traumatic Experiences Questionnaire (Vernberg, La Greca, Silverman, \& Prinstein, 1996). We also carried out qualitative interviews with some of the adolescent participants who were severally 
affected by the rainstorms. Based on the adolescents' responses and relevant psychological assessment, the questionnaire items were developed. Seven items constituted the formal questionnaire (e.g., the house was damaged by the rainstorm), and were rated on a 5-point Likert scale that ranged from 0 (completely disagree) to 4 (completely agree). Each student evaluated their exposure to daily life-threatening experiences or to disruption-loss in the week following the latest rainstorm. In this study, the questionnaire had a good internal reliability (Cronbach's $\alpha=0.83$ ).

Perceived severity of disaster. Adolescents' perceived severity of disaster was assessed using our self-developed questionnaire according to the following procedures. First, according to the contents of the assessed perception of risk involving the perceived severity of extreme events (Slovic, 1992) and the items of risk perception questionnaire related to climate change (Fu, Yang, Qin, \& Lai, 2014), we developed several items (e.g., rainstorms threaten my daily life). Next, we carried out qualitative interviews with some of the adolescent participants who were severally affected by the rainstorms according to these developed items. Based on the participants' responses and relevant psychological assessment, we changed and re-worded the items and finally developed the perceived severity of disaster questionnaire. Nine items constituted the formal questionnaire (e.g., rainstorms brought me and my family loss), and were rated on a 5point Likert scale that ranged from 0 (completely no impact) to 4 (extremely serious impact). Each student evaluated their exposure to daily life-threatening experiences or to disruption-loss in the week following the latest rainstorm. In this study, the questionnaire had a good internal reliability (Cronbach's $\alpha=0.80$ ) and validity $\left[x^{2} / \mathrm{df}=4.56, \quad \mathrm{CFI}=0.95, \quad \mathrm{TLI}=0.92, \quad \mathrm{RMSEA} \quad(90 \% \mathrm{CI})=0.061 \quad(0.050-0.073)\right.$, $\mathrm{SRMR}=0.033]$.

Rumination. Rumination was assessed by the subscale of cognitive emotion regulation 
questionnaire (Chinese revised version; Zhu, Luo, Yao, Auerbach, \& Abela, 2007). The cognitive emotion regulation questionnaire was a 36-item instrument designed to measure the following cognitive coping strategies: (1) Self-blame, (2) Acceptance, (3) Rumination, (4) Positive Refocusing, (5) Refocus on Planning, (6) Positive Reappraisal, (7) Putting things into Perspective, (8) Catastrophizing, and (9) Other-blame (Garnefski, Teerds, Kraaij, Legerstee, \& van den Kommer, 2004). All of the items were rated on a 5-point Likert scale that ranged from 0 (completely disagree) to 4 (completely agree). This questionnaire has good stability and reliability in Chinese samples (Zhu et al., 2007), and it had a good internal reliability in the current study (Cronbach's alpha=0.89). This questionnaire showed good reliability and validity in adolescents' sample following natural disaster (Zhen, Quan, Yao, \& Zhou, 2016). Considering applicability for adolescents who were severally affected by rainstorms, we carried out an interview based on the items of this questionnaire, and then reworded some of the items according to these interview results in order to ensure that all of the adolescents could understand all of the item meanings. In this study, this questionnaire also demonstrated good reliability (Cronbach's $\alpha=0.89)$ and validity $\left[\varkappa^{2} / \mathrm{df}=3.25, \mathrm{CFI}=0.91, \mathrm{TLI}=0.90, \mathrm{RMSEA}\right.$ $(90 \% \mathrm{CI})=0.049(0.046-0.051), \mathrm{SRMR}=0.066]$. We only used the rumination subscale based on our research needs, and the internal reliability of rumination subscale was acceptable here (Cronbach's $\alpha=0.71)$.

Resilience. Resilience was assessed using the Chinese version of the CD-RISC ( $\underline{\mathrm{Yu} \&}$ Zhang, 2007), a 25-item instrument that assesses the ability to cope with stress and adversity. The items are rated on a 5-point Likert scale ranging from 0 (not true at all) to 4 (true nearly all of the time). Higher scores indicate higher levels of trait resilience. A previous study demonstrated that the scale has good psychometric properties in both the general population and patient samples (Connor \& Davidson, 2003). The Chinese 
version of the CD-RISC was first translated and used by Yu and Zhang (2007) and was found to have good internal consistency, convergent validity, and discriminant validity. This scale also showed good psychometric properties in adolescents following Wenchuan earthquake (Ying, Wang, Lin, \& Chen, 2016; Ying et al., 2014). In order to assess its applicability for adolescents who were severally affected by rainstorms, we carried out an interview based on the items of this questionnaire, and then reworded some of the items according to theinterview results. This scale in the present study demonstrated good reliability (Cronbach's $\alpha=0.93$ ) and validity $\left[\varkappa^{2} / \mathrm{df}=4.36, \mathrm{CFI}=0.91\right.$, $\mathrm{TLI}=0.90, \operatorname{RMSEA}(90 \% \mathrm{CI})=0.059(0.056-0.063), \mathrm{SRMR}=0.042]$.

PTSD. PTSD was assessed using the PTSD Checklist for DSM-5(Weathers, 2013). This measure is a 20-item self-report scale that was designed to assess the occurrence and frequency of PTSD symptoms according to the Diagnostic and Statistical Manual of Mental Disorders, fifth edition, in relation to the most distressing event experienced by an individual. In the present study, all of the items were translated into Chinese. Next, we carried out an interview based on the items of this questionnaire, and then reworded some of the items according to the interview results. The respondents rated the frequencies of the symptoms during the most recent two weeks on a 4-point Likert scale that ranged from 0 (not at all/only once) to 3 (almost always or more than 5 times $a$ week). The scale had four subscales: (1) re-experiences, (2) negative cognitions and emotion alteration, (3) avoidance, and (4) hyper-arousal. An overall severity score is generated by summing the scores of the four symptom types. This scale has been previously identified as being applicable to adolescents following a natural disaster (Liu et al., 2014; Wang et al., 2015). In this sample, the scale demonstrated good reliability (Cronbach's $\alpha=0.90)$ and validity $\left[x^{2} / d f=4.08, \mathrm{CFI}=0.92, \mathrm{TLI}=0.91, \mathrm{RMSEA}(90 \% \mathrm{CI})\right.$ $=0.057(0.052-0.061), \mathrm{SRMR}=0.047]$. 


\section{Data Analysis Strategies}

Descriptive analyses were conducted for all of the measures administered. Correlations were calculated to examine the associations between major variables. Statistical analyses were conducted using Mplus 7.0 software. Before statistical analyses, we conducted an analysis of missing data on cases and variables for the main measures, respectively, and found that all of the missing rates on cases were less than $30.3 \%$, and on variables less than $0.8 \%$. Moreover, to establish the type of missing data, we used Little's Missing Completely at Random (MCAR) test. The results revealed that the data were missing at random on each of the main measures [MAR, $\chi^{2}$ (27) $=28.76, p=0.373]$. In addition, we examined the distribution of scale scores and found that skewness and kurtosis were 1.16 and 1.65 for rainstorm-related experiences, -0.14 and -0.32 for perceived severity of disasters, -0.18 and -0.07 for rumination, -0.05 and -0.14 for resilience, and 0.84 and 0.80 for PTSD. These results indicated that the scores did not substantially deviate from a normal distribution, we therefore adopted ML to handle missing data.

To evaluate the model fit, we used chi-square values, the comparative fit index (CFI), Tucker-Lewis index (TLI), normed fit index (NFI) and the root mean square error of approximation (RMSEA). A non-significant chi-square value indicated a good fit between the model and the data. The general cutoffs for accepting a model are NFI, CFI and TLI equal to or greater than 0.90, and a RMSEA of equal to or less than 0.08 (Wen, Hau, \& Marsh, 2004).

In the moderated serial multiple mediation model examination, all independent variables were first centered on their respective means to reduce multicollinearity between main effects and interaction terms, and to increase the interpretability of the coefficients on interaction terms (Cohen, Cohen, West, \& Aiken, 2013). Then, we 
followed the moderated serial multiple mediation model analysis procedures recommended by Hayes (2015) and Preacher, Rucker and Hayes (2007), and built a structure equation model (see Figure 2). Wherein, rainstorm-related experience had a direct prediction on PTSD, and an indirect prediction via perceived severity of disaster, as well as via rumination through perceived severity of disaster. Resiliency predicted perceived severity of disaster, rumination, and PTSD and the interaction term between rainstorm-related experiences and resilience also predicted perceived severity of the disaster, rumination, and PTSD.

Next, to test the significance of the indirect effect of rainstorm-related experiences on PTSD via rumination, we conducted a bias-corrected bootstrap test with a 95\% confidence interval (Gootzeit \& Markon, 2011). Also, we used a simple slope test to further examine the significance of the interaction terms.

\section{Results}

\section{Descriptive statistics and correlations among main measures}

Descriptive statistics and the correlations among the various measures are shown in Table 1. The mean levels of rainstorm-related experiences, perceived severity of disasters, rumination, resilience, and PTSD were 0.62, 2.73, 2.37, 2.34 and 0.86, respectively. Based on the diagnostic algorithm of at least one intrusion symptom, one avoidance symptom, two negative alterations in cognition and mood symptoms, and two arousal symptoms endorsed as 2 or greater, a total of 145 (15.2\%) participants were identified as probable PTSD cases. Next, Pearson correlations among the main variables were calculated. These analyses found that sex had a significant relation to rainstorm-related experiences and perceived severity of the disaster, but was not related to the other variables. Only age was associated significantly with rainstorm-related experiences and resilience. Rainstorm-related experiences were significantly, 
positively related to perceived severity of the disaster, rumination and PTSD, but was not associated with resilience. Perceived severity of the disaster was positively associated with rumination and PTSD, negatively associated with resilience. Additionally, rumination was positively related to resilience and PTSD, and resilience was negatively related to PTSD.

--Insert Table 1--

\section{Moderated serial multiple mediation analysis}

Before the moderated serial multiple mediation analysis, we carried out an examination of the direct effect of the rainstorm-related experiences on PTSD and found that the direct model fit the data better $\left[x^{2} / \mathrm{df}=1.46, C F I=0.99\right.$, TLI $=1.00$, RMSEA $(90 \% \mathrm{CI})=0.022(0.000-0.057), \mathrm{SRMR}=0.009]$. Wherein, rainstorm-related experiences positively and significantly predicted PTSD $(\beta=0.15, \mathrm{p}<0.001)$.Next, employing a moderated serial multiple mediation analysis (see Figure 2), we found that this model fit the data better $\left[\varkappa^{2} / \mathrm{df}=4.30, \quad \mathrm{CFI}=0.97, \quad \mathrm{TLI}=0.95, \quad\right.$ RMSEA $(90 \% \mathrm{CI})=0.059(0.045-0.073), \mathrm{SRMR}=0.020]$. The results of path analyses found that rainstorm-related experiences only had a significant positive prediction on the perceived severity of the disaster, but had a non-significant relation to the other variables. The perceived severity of the disaster positively predicted rumination and PTSD, and rumination positively predicted PTSD. Resilience positively predicted rumination and negatively PTSD, but demonstrated a non-significant relation with perceived severity of the disaster. The interaction term between rainstorm-related experiences and resilience negatively predicted PTSD, whereas it had a non-significant association with resilience and perceived severity of the disaster. Overall, $21 \%$ of the variance in PTSD could be explained by this model. These results indicated that rainstorm-related experiences might have a positive effect on PTSD based on one's 
perceived severity of the disaster, or by rumination via one's perceived severity of the disaster. In addition, resilience moderated the relationship between rainstorm-related experiences and PTSD, but did not moderate the relation of rainstorm-related experiences to perceived severity of the disaster and rumination.

\section{--Insert Figure 2 --}

We conducted bias-corrected bootstrap tests with a 95\% confidence interval (Gootzeit and Markon, 2011) to evaluate the significance levels of the indirect effects of rainstorm-related experiences and resilience on PTSD, and the direct effects of the interaction term between rainstorm-related experiences and resilience on perceived severity of the disaster, rumination, and PTSD, in Figure 2. Five thousand bootstrap samples were created from the original data set using random samples with replacement. The model in Figure 2 was then constructed 5000 times with these samples, to yield 5000 estimations of each indirect path coefficient. If the $95 \%$ confidence interval of an indirect path coefficient does not include 0 , it is suggested that the indirect path is indeed significant.

Table 2 shows the bias-corrected bootstrap test results. The $95 \%$ confidence intervals for the direct paths from the interaction term between rainstorm-related experiences and resilience to perceived severity of disaster and rumination included 0 , and from rainstorm-related experiences to PTSD also included 0, whereas from resilience and the interaction term between rainstorm-related experiences and resilience to PTSD did not include 0 . The $95 \%$ confidence interval for the indirect paths from rainstorm-related experiences to PTSD via perceived severity of disaster, from rainstorm-related experiences to PTSD through perceived severity of disaster via rumination, and from resilience to PTSD via rumination did not include 0 . Conversely, 
the $95 \%$ confidence intervals for the other indirect path coefficients did include 0 . These results further supported the paths in Figure 2.

--Insert Table 2--

Similarly, we used a simple slope test to further examine whether the moderating effect of resilience on the relationship between rainstorm-related experiences and PTSD was significant or not. We graphed the relation between rainstorm-related experiences and PTSD for participants whose levels of resilience were $1 \mathrm{SD}$ above or below the mean (see Figure 3). For participants $1 \mathrm{SD}$ above the mean of resilience, increased rainstorm-related experiences were not significantly associated with change in PTSD symptoms (simple slope $=0.12, t=1.32, p=0.187$ ). In contrast, for participants $1 \mathrm{SD}$ below the mean, increased rainstorm-related experiences were associated with a significant increase in PTSD symptoms (simple slope $=0.45$, $t=4.70, p<0.001)$.

-Insert Figure 3 approximately here

\section{Discussion}

To our knowledge, this is first study to examine the combined role of rainstorm-related experiences, perceived severity of disasters, rumination, and resilience in PTSD amongst adolescents following a rainstorm disaster. By using moderated serial multiple mediation analysis, the findings indicated that perceived severity of disasters and rumination fully mediated the relation of rainstorm-related experiences to PTSD, and resilience moderated the direct association between rainstorm-related experiences and PTSD.

Specifically, the findings suggest that there is a direct effect of rainstormrelated experiences on PTSD before inserting the mediators (i.e., perceived severity of 
disasters and rumination). Our findings support the shattered world assumption (JanoffBulman, 2010), and are also consistent with the findings of previous studies that that have found that traumatic experiences have a direct and positive effect on PTSD (Bokszczanin, 2007; Chen et al., 2015; Dixon, Shochet, \& Shakespeare-Finch, 2015). Furthermore, after inserting perceived severity of disasters and rumination into the relationship between rainstorm-related experiences and PTSD, the prior significant, direct association between them disappeared, which suggested that a complete mediational role in the relation of rainstorm-related experiences and PTSD. More importantly, this finding further supported our studies on PTSD amongst adolescents after the Ya'an earthquake (Wu et al., 2015; Zhou et al., 2015), and indicated that rainstorm-related experiences provide a potential predictor for PTSD. Additionally, a more important predictor or process of PTSD may be the perceived severity of disasters and the rumination following rainstorm-related experiences.

Here, we found that rainstorm-related experiences might have a one-step, indirect effect on PTSD via one's perceived severity of the disasters, which is consistent with previous studies (Besser et al., 2009; Lee et al., 2014; Lee, Joo, \& Choi, 2013; Pedrelli, Feldman, Vorono, Fava, \& Petersen, 2008) and Lazarus and Folkman'(1984) theory. That is, a stressor typically triggers a stress reaction if the stressor is perceived as a threat or if an individual feels incapable of meeting the perceived demands of a given situation (Lazarus \& Folkman,1984). Based on this theory, we believe that the rainstorm-related experiences may have elicited the adolescent participants' negative perceptions of the disaster or that they perceived the disasters as being particularly severe because it was uncontrollable (Zhou et al., 2015). The adolescents may have been more likely to perceive the rainstorm as being more threatening, and thus they felt unsafe. This loss could lead to a heightened sensitivity to threat-even minor threat, 
and in turn may have triggered a sequence of physical, emotional, and cognitive emergency responses - a state termed chronic hyperarousal (Van der Kolk, Greenberg, Orr, \& Pitman, 1988), which can cause individuals to fail to modulate their emotions and hence lead to PTSD (Bloom \& Farragher, 2010). Moreover, a greater perceived severity of the natureal disaster may have also added to the adolescents' fear and worry, thus eliciting more intrusive symptoms (Fani et al., 2012) and ultimately resulting in PTSD.

Additionally, we also found a significant multiple indirect path to PTSD from rainstorm-related experiences to perceived severity of disasters via rumination, which was consistent with our hypothesis and supported Janoff-Bulman's (2010) theory. Our results found that a higher degree of exposure to traumatic experiences led to a greater perceived severity of the disasters. Calhoun and Tedeschi (2006) suggested that for individuals who view a disaster as being more aversive, their belief system about the world as being a safe place and their sense of self-efficacy may be shattered, leading to the individuals' cognitive disequilibrium. To relieve this disequilibrium, individual need to repeatedly think about or ruminate over the traumatic events (Janoff-Bulman, 2010). Furthermore, as rumination involves repeatedly replaying the course of an experience in one's mind, focusing on how unpleasant it was ( Bruce, \& Sheline, 2015), this behavior can present traumatic cues to a traumatized individual's cognitions and, thereby, increase their intrusive symptoms, ultimately leading to PTSD. In addition, rumination may impede changes in negative appraisals of traumatic events (Ehlers, Mayou, \& Bryant, 1998), this may lead traumatized individuals to focus on the negative effects of the traumatic experiences, and thus may increase the symptoms PTSD. 
Nevertheless, we did not find a one-step, indirect effect of rainstorm-related experiences on PTSD via rumination. One possible explanation might be attributed to the non-significant, direct relation between rainstorm-related experiences and rumination, which was not consistent with previous studies (Borders et al., 2015; Chuen Yee Lo et al., 2012), but supported the findings from Zhou et al.' (2015) studies on adolescents. Here, previous studies considered rumination following a trauma as being a negative coping strategy that traumatized individual frequently use (Baschnagel et al., 2009). However, for adolescents with rainstorm-related experiences in this study, family and social groups may have been able to provide more support, which may have helped them to use more positive strategies to cope with the traumatic event. Thus, they may have been less likely to ruminate as their main coping strategy, which may explain why we found the non-significant and direct relationship between rainstorm-related experiences and rumination.

This study also found that resilience had a directly negative effect on PTSD, which was consistent with previous studies (Bonanno, 2005; Connor \& Davidson, 2003; Dai et al., 2014; Lee et al., 2014). It may be that resilience enables individuals to bounce back from adversity, and to adapt, thrive and mature in the face of adverse circumstances (Friborg, Hjemdal, Rosenvinge, \& Martinussen, 2003; Liu, Wang, Zhou,

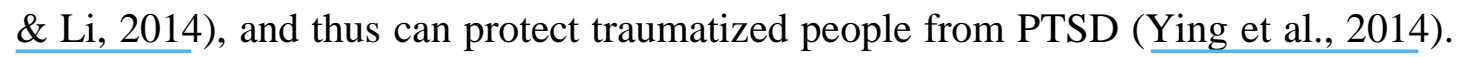
Nevertheless, an unexpected but interesting finding was the significantly positive association between resilience and rumination. This was inconsistent with previous studies (Calhoun \& Tedeschi, 2006; Thanoi, Phancharoenworakul, Thompson, Panitrat, \& Nityasuddhi, 2010; Tugade \& Fredrickson, 2004). A potential explanation may be that adolescents have immature cognitive and emotional capabilities., Although resilience can lead to more optimistic thinking (Tugade \& Fredrickson, 2004), when 
this thinking focuses on traumatic experiences, traumatic cues may also intrude into the cognitive world of the traumatized individuals, and thus may result in intrusive rumination.

Consistent with previous studies (Dai et al., 2014; Lee et al., 2014), we found that resilience could buffer the effect of rainstorm-related experiences on PTSD, but resilience only moderated the direct association between rainstorm-related experiences and PTSD. Specifically, for participants in the higher resiliency group, increased rainstorm-related experiences were not significantly associated with a change in PTSD symptoms. In contrast, for participants in the lower resiliency group, increased rainstorm-related experiences were associated with a significant increase in PTSD. Resilient adolescents displayed a greater hardiness, as well as higher rates of selfesteem, optimism, and self-enhancement than adolescents with lower levels of resiliency (Bonanno, 2005, 2008). Resilient adolescents displayed greater resistance to the negative effects of adversity (Benight \& Bandura, 2004), and had more optimistic attitudes in the face of adversity (Liu et al., 2014). Some of the participants even considered the adversity to be a normal life event, such that their reactions remained stable during and after their exposure to the trauma (Bonanno, 2008), and in this way the rainstorm-related experiences may have non-significant effects on developing PTSD. For adolescents with less resilience, they displayed lower levels of optimism, self-esteem, and hope at baseline (Besser, Zeigler-Hill, Weinberg, Pincus, \& Neria, 2015), thereby making them more likely to be susceptible to the effects of the traumatic experiences (Pietrzak et al., 2009). Once they experienced a highly stressful event, (i.e., the rainstorm disaster), this experience may have further reduced their levels of selfesteem, hope, and aggravated their negative attitude, leading to even higher levels of distress. 
Several design and measurement limitations must be acknowledged. First, the participants were not randomly selected but rather represent a convenience sample due to the nature of the investigation. As a result, one cannot eliminate the possibility of a sampling bias. Second, all variables were measured by self-report, and so associations between the main measures might be affected by common-method variance. Third, the study was conducted with a sample of adolescents after the rainstorm in China, so generalizations to other individuals with different traumatic experiences must be done with caution. Additionally, the rainstorm-related experiences and perceived severity of the disaster were assessed by using our own self-developed questionnaire. While the developing process of these two questionnaires was based on previous studies and interviews with adolescents, it still lacked the standardized and strict procedures typically used in building a questionnaire with good reliability and validity. Therefore, further development of standardized questionnaires to carry out further research on this topic would be recommended.

Despite these limitations, this study indicated that the effect of traumatic experiences on PTSD may be realized via multiple indirect paths, further suggesting that exposure to a trauma is a very objective experience, and its impact on individuals' posttraumatic reactions largely depends on their own cognitive and emotional processes of the traumatic events and their consequences (Zhou et al., 2015). In addition, this study suggests that although resilience in adolescents can buffer PTSD following traumatic exposure, resilient adolescents may also engage in rumination because of their immature cognitive and emotional capability. Our results may, therefore, contribute to the theoretical and empirical studies on PTSD following trauma and resilience amongst adolescents. From an intervention and health-enhancement perspective, this study also highlights important implications for adolescents after a 
traumatic rainstorm. Clinical efforts should focus on decreasing their perceived severity of the event and the levels of post-traumatic rumination, thereby helping to distract their focus from negative aspects of the rainstorm. Additionally, school psychologists or parents should focus on the cultivation of hardiness, self-esteem, and self-enhancement amongst adolescent children, and encourage them to acknowledge any reminders of the traumatic event and any negative emotions they may experience to others in order to mitigate the possible negative effects of the trauma. 


\section{References}

Agustini, E. N., Asniar, I., \& Matsuo, H. (2011). The prevalence of long - term post traumatic stress symptoms among adolescents after the tsunami in Aceh. Journal of Psychiatric and Mental Health Nursing, 18, 543-549.

Baschnagel, J. S., Gudmundsdottir, B., Hawk, L. W., \& Beck, J. G. (2009). Posttrauma symptoms following indirect exposure to the September 11th terrorist attacks: The predictive role of dispositional coping. Journal of Anxiety Disorders, 23, 915-922.

Baumeister, R. F., Gailliot, M., DeWall, C. N., \& Oaten, M. (2006). Self-Regulation and personality: How interventions increase regulatory success, and how depletion moderates the effects of traits on behavior. Journal of Personality, 74, 1773-1802.

Benight, C. C., \& Bandura, A. (2004). Social cognitive theory of posttraumatic recovery: The role of perceived self-efficacy. Behaviour Research and Therapy, 42, 1129-1148.

Bensimon, M. (2012). Elaboration on the association between trauma, PTSD and posttraumatic growth: The role of trait resilience. Personality and Individual Differences, 52, 782-787.

Besser, A., Neria, Y., \& Haynes, M. (2009). Adult attachment, perceived stress, and PTSD among civilians exposed to ongoing terrorist attacks in Southern Israel. Personality and Individual Differences, 47, 851-857.

Besser, A., \& Priel, B. (2010). Personality vulnerability, low social support, and $\underline{\text { maladaptive cognitive emotion regulation under ongoing exposure to terrorist }}$ attacks. Journal of Social and Clinical Psychology, 29, 166-201.

Besser, A., Zeigler-Hill, V., Weinberg, M., Pincus, A. L., \& Neria, Y. (2015). 
Intrapersonal resilience moderates the association between exposure-severity and PTSD symptoms among civilians exposed to the 2014 Israel-Gaza conflict. Self and Identity, 14, 1-15.

Blackburn, L., \& Owens, G. P. (2016). Rumination, resilience, and posttraumatic stress disorder symptom severity among veterans of Iraq and Afghanistan. Journal of Aggression, Maltreatment \& Trauma, 25, 197-209.

Blanchard, E. B., Hickling, E. J., Mitnick, N., Taylor, A. E., Loos, W. R., \& Buckley, T. C. (1995). The impact of severity of physical injury and perception of life threat in the development of post-traumatic stress disorder in motor vehicle accident victims. Behaviour Research and Therapy, 33, 529-534.

Bloom, S. L., \& Farragher, B. (2010). Destroying sanctuary: The crisis in human service delivery systems: Oxford University Press.

Bokszczanin, A. (2007). PTSD symptoms in children and adolescents 28 months after a flood: Age and gender differences. Journal of Traumatic Stress, 20, 347-351.

Bomyea, J., \& Lang, A. J. (2016). Accounting for intrusive thoughts in PTSD:

Contributions of cognitive control and deliberate regulation strategies. Journal of Affective Disorders, 192, 184-190.

Bonanno, G. A. (2004). Loss, trauma, and human resilience: have we underestimated the human capacity to thrive after extremely aversive events? American Psychologist, 59, 20-28.

Bonanno, G. A. (2005). Clarifying and Extending the Construct of Adult Resilience. American Psychologist, 60, 265-267.

Bonanno, G. A. (2008). Loss, trauma, and human resilience: Have we underestimated the human capacity to thrive after extremely aversive events? Psychological Trauma: Theory, Research, Practice, and Policy, 1, 101-113. 
Borders, A., Rothman, D. J., \& McAndrew, L. M. (2015). Sleep problems may mediate associations between rumination and PTSD and depressive symptoms among OIF/OEF veterans. Psychological Trauma: Theory, Research, Practice, and Policy, 7, 76-84.

Calhoun, L. G., \& Tedeschi, R. G. (2006). The foundations of posttraumatic growth: An expanded framework. In L. G. Calhoun \& R. G. Tedeschi (Eds.), Handbook of posttraumatic growth (pp. 1-23). Mahwah, NJ: Erlbaum.

Cao, X., Wang, L., Cao, C., \& Zhang, J. (2015). The phenotypic model of posttraumatic stress disorder symptom dimensionality[Chinese]. Journal of Beijing Normal University (Social Sciences),(6), 87-99.

Cernvall, M., Skogseid, E., Carlbring, P., Ljungman, L., Ljungman, G., \& von Essen, L. (2016). Experiential avoidance and rumination in parents of children on cancer treatment: relationships with posttraumatic stress symptoms and symptoms of depression. Journal of Clinical Psychology in Medical Settings, $23,67-76$.

Chen, L., Tan, H., Cofie, R., Hu, S., Li, Y., Zhou, J., . . Liu, A. (2015). Prevalence and determinants of chronic post-traumatic stress disorder after floods. Disaster Medicine and Public Health Preparedness, 9, 504-508.

Chuen Yee Lo, B., Lau, S., Cheung, S.H., \& Allen, N. B. (2012). The impact of rumination on internal attention switching. Cognition \& Emotion, 26, 209-223.

Cohen, J., Cohen, P., West, S. G., \& Aiken, L. S. (2013). Applied multiple regression/correlation analysis for the behavioral sciences. Mahwah, NJ: Routledge.

Connor, K. M., \& Davidson, J. R. T. (2003). Development of a new resilience scale: The Connor-Davidson resilience scale (CD-RISC). Depression and Anxiety, 
18, 76-82.

Dai, Y., Lei, M., Zhou, X., Yao, M., Jiang, J., Chen, X., \& Liu, Y. (2014). The effect of trauma expose on posttraumatic stress disorder after the Wenchuan earthquake: The role of resilience as a moderator[Chinese]. Psychological Development and Education 30, 61-67.

Delahanty, D. L., Raimonde, A. J., Spoonster, E., \& Cullado, M. (2003). Injury severity, prior trauma history, urinary cortisol levels, and acute PTSD in motor vehicle accident victims. Journal of Anxiety Disorders, 17, 149-164.

Dixon, K. M., Shochet, I. M., \& Shakespeare-Finch, J. (2015). Stress during the rebuilding phase influenced mental health following two Queensland flood disasters more than the event itself. Paper presented at the Australian and New Zealand Disaster and Emergency Management Conference, Broadbeach, Gold Coast, Q1d.

Ehlers, A., Mayou, R. A., \& Bryant, B. (1998). Psychological predictors of chronic posttraumatic stress disorder after motor vehicle accidents. Journal of Abnormal Psychology, 107, 508-519.

Eth, S. (2008). PTSD in children and adolescents. Washington, DC: American Psychiatric Pub.

Fan, F., Zhang, Y., Yang, Y., Mo, L., \& Liu, X. (2011). Symptoms of posttraumatic stress disorder, depression, and anxiety among adolescents following the 2008 Wenchuan earthquake in China. Journal of Traumatic Stress, 24, 44-53.

Fani, N., Tone, E. B., Phifer, J., Norrholm, S. D., Bradley, B., Ressler, K. J., . . . Jovanovic, T. (2012). Attention bias toward threat is associated with exaggerated fear expression and impaired extinction in PTSD. Psychological Medicine, 42, 533-543. 
Fincham, D. S., Altes, L. K., Stein, D. J., \& Seedat, S. (2009). Posttraumatic stress disorder symptoms in adolescents: Risk factors versus resilience moderation. Comprehensive Psychiatry, 50, 193-199.

Freedy, J. R., Resnick, H. S., \& Kilpatrick, D. G. (1992). Conceptual framework for evaluating disaster impact: Implications for clinical intervention. In L. S. Austin (Ed.), Responding to disaster: A guide for mental health professionals (pp. 6-14). Washington, DC: American Psychiatric Press.

Friborg, O., Hjemdal, O., Rosenvinge, J. H., \& Martinussen, M. (2003). A new rating scale for adult resilience: what are the central protective resources behind healthy adjustment? International Journal of Methods in Psychiatric Research, 12, 65-76.

Fu, Y., Yang, S., Qin, Q., \& Lai, F. (2014). The structure of risk perception related to climate change-- An exploratory study[Chinese] Psychological Research, 7, 5156.

Furr, J. M., Comer, J. S., Edmunds, J. M., \& Kendall, P. C. (2010). Disasters and youth: a meta-analytic examination of posttraumatic stress. Journal of Consulting and Clinical Psychology, 78, 765-780.

Galea, S., Nandi, A., \& Vlahov, D. (2005). The epidemiology of post-traumatic stress disorder after disasters. Epidemiologic Reviews, 27, 78-91.

Garnefski, N., Teerds, J., Kraaij, V., Legerstee, J., \& van den Kommer, T. (2004). Cognitive emotion regulation strategies and depressive symptoms: Differences between males and females. Personality and Individual Differences, 36, 267276.

Girelli, S. A., Resick, P. A., Marhoefer-Dvorak, S., \& Hutter, C. K. (1986). Subjective distress and violence during rape: Their effects on long-term fear. Violence and 
Victims, 1, 35-46.

Gootzeit, J., \& Markon, K. (2011). Factors of PTSD: Differential specificity and external correlates. Clinical Psychology Review, 31, 993-1003.

Grubaugh, A. L., Zinzow, H. M., Paul, L., Egede, L. E., \& Frueh, B. C. (2011). Trauma exposure and posttraumatic stress disorder in adults with severe mental illness: a critical review. Clinical Psychology Review, 31, 883-899.

Hayes, A. F. (2015). An index and test of linear moderated mediation. Multivariate Behavioral Research, 50, 1-22.

Hu, E., Koucky, E. M., Brown, W. J., Bruce, S. E., \& Sheline, Y. I. (2015). The role of rumination in elevating perceived stress in posttraumatic stress disorder. Journal of Interpersonal Violence, 29,1953-1962.

Jakšić, N., Brajković, L., Ivezić, E., Topić, R., \& Jakovljević, M. (2012). The role of personality traits in posttraumatic stress disorder (PTSD). Psychiatria Danubina, 24(3.), 256-266.

Janoff-Bulman, R. (2010). Shattered assumptions. New York: Simon and Schuster.

Joseph, S., Williams, R., \& Yule, W. (1997). Understanding post-traumatic stress: A psychosocial perspective on PTSD and treatment. Chichester,West Sussex,England: Wiley.

Jovanovic, T., Norrholm, S. D., Fennell, J. E., Keyes, M., Fiallos, A. M., Myers, K. M., . . Duncan, E. J. (2009). Posttraumatic stress disorder may be associated with impaired fear inhibition: relation to symptom severity. Psychiatry Research, 167, 151-160.

Kemp, A., Rawlings, E. I., \& Green, B. L. (1991). Post-traumatic stress disorder (PTSD) in battered women: A shelter sample. Journal of Traumatic Stress, 4, $\underline{137-148 .}$ 
Lazarus, R., \& Folkman, S. (1984). Stress Appraisal and Coping. New York: Springer.

Lee, J.S., Ahn, Y.S., Jeong, K.S., Chae, J.H., \& Choi, K.S. (2014). Resilience buffers the impact of traumatic events on the development of PTSD symptoms in firefighters. Journal of Affective Disorders, 162, 128-133.

Lee, J. S., Joo, E. J., \& Choi, K. S. (2013). Perceived stress and self-esteem mediate the effects of work-related stress on depression. Stress and Health, 29, 75-81.

Liu, P., Wang, L., Cao, C., Wang, R., Zhang, J., Zhang, B., . . Fan, G. (2014). The underlying dimensions of DSM-5 posttraumatic stress disorder symptoms in an epidemiological sample of Chinese earthquake survivors. Journal of Anxiety Disorders, 28, 345-351.

Liu, Y., Wang, Z., Zhou, C., \& Li, T. (2014). Affect and self-esteem as mediators between trait resilience and psychological adjustment. Personality and Individual Differences, 66, 92-97.

Ma, X., Liu, X., Hu, X., Qiu, C., Wang, Y., Huang, Y., . . Li, T. (2011). Risk indicators for post-traumatic stress disorder in adolescents exposed to the 5.12 Wenchuan earthquake in China. Psychiatry Research, 189, 385-391.

McKeever, V. M., \& Huff, M. E. (2003). A diathesis-stress model of posttraumatic stress disorder: Ecological, biological, and residual stress pathways. Review of General Psychology, 7, 237-250.

Michl, L. C., McLaughlin, K. A., Shepherd, K., \& Nolen-Hoeksema, S. (2013). Rumination as a mechanism linking stressful life events to symptoms of depression and anxiety: longitudinal evidence in early adolescents and adults. Journal of Abnormal Psychology, 122, 339-352.

Nabizadeh, F., \& Mahdavi, A. (2016). Relationship between Hardiness and Marital Satisfaction in Women with Breast Cancer. Archives of Breast Cancer, 3, 92- 
96.

Nolen-Hoeksema, S., Wisco, B. E., \& Lyubomirsky, S. (2008). Rethinking rumination. Perspectives on Psychological Science, 3, 400-424.

Nygaard, E., \& Heir, T. (2012). World assumptions, posttraumatic stress and quality of life after a natural disaster: A longitudinal study. Health and Quality of Life Outcomes, 10, 76-83.

Park, C. L. (2010). Making sense of the meaning literature: an integrative review of $\underline{\text { meaning making and its effects on adjustment to stressful life events. }}$ Psychological Bulletin, 136, 257-301.

Pedrelli, P., Feldman, G. C., Vorono, S., Fava, M., \& Petersen, T. (2008). Dysfunctional attitudes and perceived stress predict depressive symptoms severity following antidepressant treatment in patients with chronic depression. Psychiatry Research, 161, 302-308.

Pietrzak, R. H., Johnson, D. C., Goldstein, M. B., Malley, J. C., \& Southwick, S. M. (2009). Psychological resilience and postdeployment social support protect against traumatic stress and depressive symptoms in soldiers returning from Operations Enduring Freedom and Iraqi Freedom. Depression and Anxiety, 26, $745-751$.

Ponnamperuma, T., \& Nicolson, N. A. (2016). Negative trauma appraisals and PTSD symptoms in Sri Lankan adolescents. Journal of Abnormal Child Psychology, 44, 245-255.

Preacher, K. J., Rucker, D. D., \& Hayes, A. F. (2007). Addressing moderated mediation hypotheses: Theory, methods, and prescriptions. Multivariate Behavioral Research, 42, 185-227.

Slovic, P. (1992). Perception of risk: Reflections on the psychometric paradigm. In S. 
Krimsky \& D. Golding (Eds.), Social Theories of Risk (pp. 117--152).

London: Praeger.

Scher, C. D., Ingram, R. E., \& Segal, Z. V. (2005). Cognitive reactivity and vulnerability: Empirical evaluation of construct activation and cognitive diatheses in unipolar depression. Clinical Psychology Review, 25, 487-510.

Solomon, Z., Mikulincer, M., \& Hobfoll, S. E. (1987). Objective versus subjective measurement of stress and social support: combat-related reactions. Journal of Consulting and Clinical Psychology, 55, 577-583.

Tedeschi, R. G., \& Calhoun, L. G. (2004). Posttraumatic growth: Conceptual foundations and empirical evidence. Psychological Inquiry, 15, 1-18.

Thanoi, W., Phancharoenworakul, K., Thompson, E. A., Panitrat, R., \& Nityasuddhi, D. (2010). Thai adolescent suicide risk behaviors: testing a model of negative life events, rumination, emotional distress, resilience and social support. Pacific Rim International Journal of Nursing Research, 14, 187-202.

Trickey, D., Siddaway, A. P., Meiser-Stedman, R., Serpell, L., \& Field, A. P. (2012). A meta-analysis of risk factors for post-traumatic stress disorder in children and adolescents. Clinical Psychology Review, 32, 122-138.

Tugade, M. M., \& Fredrickson, B. L. (2004). Resilient individuals use positive emotions to bounce back from negative emotional experiences. Journal of Personality and Social Psychology, 86, 320-333.

Van der Kolk, B. A., Greenberg, M. S., Orr, S. P. e., \& Pitman, R. K. (1988). Endogenous opioids, stress induced analgesia, and posttraumatic stress disorder. Psychopharmacology Bulletin, 25, 417-421.

Vernberg, E. M., La Greca, A. M., Silverman, W. K., \& Prinstein, M. J. (1996). Prediction of posttraumatic stress symptoms in children after Hurricane 
Andrew. Journal of Abnormal Psychology, 105, 237-248.

Wagnild, G. (2009). A review of the Resilience Scale. Journal of nursing measurement, 17(2), 105-113.

Wang, L., Zhang, L., Armour, C., Cao, C., Qing, Y., Zhang, J., . . Zhao, Z. (2015). Assessing the underlying dimensionality of DSM-5 PTSD symptoms in Chinese adolescents surviving the 2008 Wenchuan earthquake. Journal of Anxiety Disorders, 31, 90-97.

Weathers, F.W., (2013).The PTSD Checklist for DSM-5 (PCL-5): Development and initial psychometric analysis. Paper presented at the 29th Annual meeting of the International Society for Traumatic Stress Studies, Philadelphia, PA.

Wen, Z. L., Hau, K. T., \& Marsh, H. W. (2004). Structual equation model testing: Cutoff criteria for goodness of fit indices and Chi-square test[Chinese]. Acta Psychologica Sinica, 36, 186-194.

Wingo, A. P., Wrenn, G., Pelletier, T., Gutman, A. R., Bradley, B., \& Ressler, K. J. (2010). Moderating effects of resilience on depression in individuals with a history of childhood abuse or trauma exposure. Journal of Affective Disorders, $126,411-414$.

Wu, X., Zhou, X., Lin, C., \& Chen, J. (2015). The influencing mechanism and mental guidance of adolescents' traumatic reaction[Chinese]. Psychological Development and Education, 31, 117-127.

Ying, L., Chen, C., Lin, C., Greenberger, E., Wu, X., \& Jiang, L. (2015). The relationship between posttraumatic stress symptoms and suicide ideation among child survivors following the Wenchuan earthquake. Suicide and LifeThreatening Behavior, 45, 230-242.

Ying, L., Wang, Y., Lin, C., \& Chen, C. (2016). Trait resilience moderated the 
relationships between PTG and adolescent academic burnout in a post-disaster context. Personality and Individual Differences, 90, 108-112.

Ying, L., Wu, X., Lin, C., \& Jiang, L. (2014). Traumatic severity and trait resilience as predictors of posttraumatic stress disorder and depressive symptoms among adolescent survivors of the Wenchuan earthquake. PLOS ONE, 9, e89401.

Yu, X., \& Zhang, J. (2007). Factor analysis and psychometric evaluation of the Connor-Davidson resilience scale (CD-RISC) with Chinese people. Social Behavior and Personality: an International Journal, 35, 19-30.

Zhen, R., Quan, L., Yao, B., \& Zhou, X. (2016). Understanding the relationship between $\underline{\text { rainstorm-related experiences and PTSD among Chinese adolescents after }}$ rainstorm disaster: The roles of rumination and social support. Frontiers in Psychology, 7, 1407.

Zhou, X., \& Wu, X. (2016). The relationship between rumination, posttraumatic stress disorder, and posttraumatic growth among Chinese adolescents after earthquake: A longitudinal study. Journal of Affective Disorders, 193, 242248.

Zhou, X., Wu, X., Yuan, X., Chen, J., \& Chen, Q. (2015). The role of core beliefs challenge, subjective fear and intrusive rumination in associations between the severity of traumatic expose and posttraumatic stress disorder in adolescent survivors after Yaan earthquake[Chinese]. Acta Psychologica Sinica, 47, 455465.

Zhu, X., Luo, F., Yao, S., Auerbach, R. P., \& Abela, J. R. Z. (2007). Reliability and validity of the Cognitive Emotion Regulation Questionnaire-Chinese version[Chinese]. Chinese Journal of Clinical Psychology, 15, 121-124. 
Table 1 Means, standard deviations, and correlations among main variables

\begin{tabular}{|c|c|c|c|c|c|c|c|}
\hline & $\mathrm{M} \pm \mathrm{SD}$ & 1 & 2 & 3 & 4 & 5 & 6 \\
\hline 1.Sex & -- & 1.00 & & & & & \\
\hline 2.Age & $14.78 \pm 1.70$ & 0.04 & 1.00 & & & & \\
\hline 3.Rainstorm-related experiences & $0.62 \pm 0.61$ & $0.14^{* *}$ & 0.05 & 1.00 & & & \\
\hline 4. Perceived severity & $2.73 \pm 0.73$ & $0.07^{*}$ & $0.15^{* *}$ & $0.43^{* *}$ & 1.00 & & \\
\hline 5. Rumination & $2.37 \pm 0.85$ & -0.01 & 0.04 & $0.07^{*}$ & $0.09^{* *}$ & 1.00 & \\
\hline 6. Resilience & $2.34 \pm 0.67$ & -0.01 & $-0.11^{* *}$ & -0.05 & $-0.07^{*}$ & $0.23^{* *}$ & 1.00 \\
\hline 7. PTSD & $0.86 \pm 0.52$ & -0.02 & -0.04 & $0.15^{* *}$ & $0.25^{* *}$ & $0.28^{* *}$ & $-0.13^{* *}$ \\
\hline
\end{tabular}

Note: Perceived severity=perceived severity of disaster.

**. Correlation is significant at the 0.01 level (2-tailed);

*. Correlation is significant at the 0.05 level (2-tailed). 
Table 2 Bias-corrected Bootstrap test

Paths

Standardized $\beta(S E) \quad$ Standardized 95\%

Low High

\section{Direct paths}

Experiences-PTSD

$\begin{array}{ccc}0.03(0.04) & -0.03 & 0.09 \\ -0.21(0.04)^{* * *} & -0.28 & -0.15 \\ -0.09(0.05)^{*} & -0.16 & -0.01 \\ 0.03(0.04) & -0.03 & 0.08 \\ -0.01(0.03) & -0.07 & 0.04\end{array}$

Resilience-PTSD

Interaction term -PTSD

Interaction term - perceived severity

Interaction term - rumination

$-0.01(0.03)$

$\begin{array}{ccc}0.09(0.02)^{* * *} & 0.07 & 0.12 \\ 0.02(0.01) & -0.01 & 0.04 \\ 0.01(0.006)^{*} & 0.003 & 0.02 \\ -0.01(0.01) & -0.02 & 0.002 \\ 0.08(0.02)^{* * *} & 0.06 & 0.10\end{array}$

Resilience -rumination -PTSD

$-0.001(0.04)$

$-0.003 \quad 0.001$

Resilience -perceived severity-rumination -PTSD

Note: Experiences $=$ Rainstorm-related experiences, perceived severity $=$ perceived

severity of disaster, Interaction term= Interaction term between rainstorm-related

experiences and resilience.

***. Path is significant at the 0.001 level (2-tailed);

**. Path is significant at the 0.01 level (2-tailed);

*. Path is significant at the 0.05 level (2-tailed). 
Figure 1. The concept figure of proposed moderated serial multiple mediation model

Note: Experiences represent rainstorm-related experiences, perceived severity represents perceived severity of disaster.

Figure 2. Moderated serial multiple mediation model

Note: Experiences and Exper represent rainstorm-related experiences, perceived severity represents perceived severity of disaster, NCAEA represents negative cognition and emotion alteration of PTSD;

***. Path is significant at the 0.001 level (2-tailed);

**. Path is significant at the 0.01 level (2-tailed);

*. Path is significant at the 0.05 level (2-tailed).

Figure 3. Relationship between rainstorm-related experiences and PTSD at different levels of resilience

Please see this article: http://dx.doi.org/10.1016/j.apnu.2017.06.003 